\title{
Características del fruto de vainilla verde y beneficiada cosechados a diferente edad
}

Characteristics of green and cured vanilla fruit harvested at different ages
Juan Vargas-Hernandez ${ }^{1,2}$ (D) , Ma. de Lourdes Arévalo-Galarza ${ }^{1^{*}}$ (D) , Adriana Delgado-Alvarado ${ }^{3}$ (D) , Cecilia Osorio-García ${ }^{1}$ (D) ; Ángel Villegas-Monter ${ }^{1}$ (D) , Hilda Araceli Zavaleta-Mancera ${ }^{1}$

\footnotetext{
${ }^{1}$ Colegio de Postgraduados-Campus Montecillo, Carretera México-Texcoco km 36.5, Montecillo, Texcoco, 56230, Estado de México, México.

${ }^{2}$ Instituto Nacional de Investigaciones Forestales, Agrícolas y Pecuarias; Centro de Investigación Regional del Noreste, Campo Experimental San Luis, Ejido Palma de la Cruz Soledad de Graciano Sánchez, 78431, San Luis Potosí, México.

${ }^{3}$ Colegio de Postgraduados-Campus Puebla, Boulevard Forjadores de Puebla núm. 25, Santiago Momoxpan, San Pedro Cholula, 72760, Puebla, México.
}

*Autor para correspondencia: larevalo@colpos.mx

Fecha de recepción:

9 de febrero de 2021

Fecha de aceptación:

13 de agosto de 2021

\section{Disponible en línea:}

21 de diciembre de 2021

Este es un artículo en acceso abierto que se distribuye de acuerdo a los términos de la licencia Creative Commons.

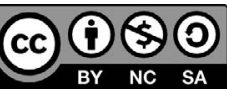

Reconocimiento-

NoComercia-

CompartirIgual 4.0

Internacional

\section{RESUMEN}

En México el fruto de vainilla (Vanilla planifolia Jacks. ex Andrews) se cosecha a partir de las 32 semanas después de la polinización (SDP). Sin embargo, los altos precios del fruto han llevado a cosecharlo poco después de las 20 SDP con efectos negativos en la calidad del fruto beneficiado. Por ello, con la finalidad de proporcionar información a los productores y optar por cosechar en el mejor momento se evaluaron las características de calidad de fruto verde y beneficiado de 20, 24, 28 y 32 SDP. Los resultados mostraron que los frutos de 28 SDP tuvieron mayor acumulación de azúcares totales (25.8 \%), que se redujeron drásticamente en el fruto beneficiado (4.39 \%). El contenido de macro y micronutrientes, incrementó conforme avanzó la edad del fruto, particularmente a partir de 24 SDP, siendo Ca, K, Mn y Fe los más abundantes.

\section{PALABRAS CLAVE}

Polinización, curado, contenido de azúcares, macronutrientes, micronutrientes

\section{ABSTRACT}

In Mexico, vanilla fruit (Vanilla planifolia Jacks. ex Andrews) is harvested at 32 weeks after pollination (WAP). However, the high prices of the fruit have led to an early harvest shortly after 20 WAP with negative effects on the quality of the cured fruit. Therefore, in order to provide information to growers and find the best time for harvest, this project evaluated the quality characteristics of green and cured fruit from 20, 24, 28 and 32 WAP. The results showed that the 28 WAP fruits had higher accumulation of total sugars $(25.8 \%)$, which were drastically reduced in the cured fruit (4.39\%). The content of macro and micronutrients increased as the age of the fruit advanced, particularly from 24 WAP, with $\mathrm{Ca}, \mathrm{K}, \mathrm{Mn}$ and Fe being the most abundant.

\section{KEYWORDS}

Pollination, curing, sugar content, macronutrients, micronutrients 


\section{INTRODUCCIÓN}

El fruto de vainilla (Vanilla planifolia Jacks. ex Andrews) es la segunda especia más cara del mundo, después del azafrán, con precios de US $\$ 600 \mathrm{~kg}^{-1}$ y $\$ 18,000$ $\mathrm{kg}^{-1}$, respectivamente (Hooker 2017; Núñez-Torrón 2020). Desde la polinización hasta la cosecha, el fruto requiere de 32 a 36 semanas $y$, posteriormente, se somete a un proceso de beneficio (curado) con el fin de que desarrolle sus características óptimas de sabor y aroma (Havkin-Frenkel et al. 2004).

El fruto de vainilla, botánicamente, es una cápsula (Font-Quer 1953), que se desarrolla a partir de la primera semana después de la polinización, para alcanzar su máximo crecimiento (largo y ancho) entre las 7 y 10 semanas (Van Dyk et al. 2014). El fruto es dehiscente, con peso de 10 a $20 \mathrm{~g}$ (dependiendo el origen) (Brillouet et al. 2010; Cervantes et al. 2018), longitud entre $18-20 \mathrm{~cm}$, diámetro de 0.10 a 0.15 cm y ancho de 0.2 a $0.5 \mathrm{~cm}$ (Bory 2007; Cervantes et al. 2018). Su color es verde brillante cuando está inmaduro y verde-amarillo cuando es maduro, para después tornarse café en la senescencia (Fouché y Jouve 1999; Hernández y Lubinsky 2011). Al madurar, la parte carnosa exterior del fruto se pigmenta a verde, mientras que la porción interior que rodea la cavidad central es amarilla (Odoux y Brillouet 2009).

Al momento de la cosecha, el fruto de vainilla carece de aroma, por lo cual es necesario el proceso de beneficio. Puesto que el fruto verde contiene precursores del aroma en forma glucosilada, el beneficio o curado no sólo deshidrata el fruto, sino que promueve cambios en la microestructura y reacciones enzimáticas, que conducen a la formación de aromas y sabores; en este sentido, la enzima $\beta$-glucosidasa es la más importante (Dignum et al. 2004).

Debido al alto costo de la vainilla, en muchas regiones vainilleras se presentan problemas de robo del fruto, lo cual provoca que los productores tomen la decisión de cosechar con antelación, incluso poco después de la semana 20 después de polinización (Núñez-Torrón 2020). El estado de madurez del fruto es uno de los factores que determina la calidad de la vainilla beneficiada (Van Dyk et al. 2014). Cuando se beneficia un fruto inmaduro, el periodo de deshidratación se alarga, lo que da como resultado un fruto delgado con poca flexibilidad, color ocre $y$ líneas rojizas que no cumplen con la norma mexicana NOM-182-SCFI-2011 (SE 2011). Por lo anterior, esta investigación tuvo el objetivo de conocer las características de calidad del fruto con diferente edad (20, 24, 28 y 32 semanas después de la polinización SDP -), tanto verde como beneficiado, con la finalidad de proporcionar a los productores información que les ayude a tomar la decisión de optar por la mejor edad de cosecha de la vainilla.

\section{Materiales y Métodos}

El estudio se llevó a cabo en una plantación comercial de tres años, que se ubica en Mahuajco, Coxcatlán,

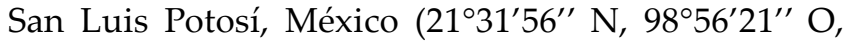
$306 \mathrm{msnm})$, con temperatura media anual de $24.5^{\circ} \mathrm{C}$ (SMN 2019) y clima semicálido húmedo $(\mathrm{A}) \mathrm{C}(\mathrm{m})(\mathrm{w})$ (García 2005).

El periodo de floración inició en abril de 2019. En antesis se seleccionaron 40 matas de vainilla al azar y se polinizaron manualmente 400 flores durante un periodo de siete días. Con base en el periodo de desarrollo del fruto hasta la cosecha se establecieron cuatro fechas de muestreo, correspondientes a 20, 24, 28 y 32 semanas después de la polinización (SDP).

Los muestreos se llevaron a cabo del 8 de septiembre al 8 de diciembre de 2019. Para cada fecha de muestreo se tomaron 30 frutos: 15 para evaluar la calidad del fruto verde y 15 para fruto beneficiado. El proceso de beneficio para cada lote de fruto siguió el método tradicional (Xochipa-Morante et al. 2016) y se realizó en la casa beneficiadora denominada "30 Soles", ubicada en la localidad Jalpilla Viejo, Axtla de Terrazas, San Luis Potosí, México. El beneficio inició con el matado del fruto de vainilla con inmersión en agua caliente (65 a $70{ }^{\circ} \mathrm{C}$; 2 a $3 \mathrm{~min}$ ); posteriormente, los frutos se acomodaron en cajones de madera para el sudado $\left(45\right.$ a $65^{\circ} \mathrm{C}$ ) por 12 a $24 \mathrm{~h}$. Después, se sacaron y se expusieron al Sol hasta alcanzar 45 ${ }^{\circ} \mathrm{C}$, y nuevamente se guardaron en los cajones para el sudado. Esta operación cíclica se repitió 25 veces, hasta obtener un fruto sin daños físicos, flexible, con un color entre café rojizo y café oscuro brillante, con entre 25 y $30 \%$ de humedad y aroma agradable (Dunphy y Bala 2011). 


\section{Variables evaluadas}

En los frutos verdes y beneficiados se midieron las siguientes variables: peso (g), dimensiones (longitud, diámetro, ancho); porcentaje de materia seca y humedad; contenido de azúcares (glucosa, fructosa y sacarosa); análisis nutricional (macro y microelementos). El peso de los frutos se midió con una balanza digital (modelo SI-2000S, Setra Systems ${ }^{\circledR}$, Boxborough, MA, Estados Unidos) (0.01 g). Las dimensiones longitud $(\mathrm{mm})$, ancho $(\mathrm{mm})$ y grosor $(\mathrm{mm})$ de las vainas se midieron con un vernier digital (modelo CD-AX/APX Series, Mitutoyo ${ }^{\circledR}$, Japón).

Para determinar el contenido de materia seca (MS) se pesaron $500 \mathrm{mg}$ de fruto y se secaron en una estufa de aire (modelo Imperial II, Lab-Line Instruments ${ }^{\circledR}$, Illinois, Estados Unidos), con aire forzado a $80{ }^{\circ} \mathrm{C}$ por $48 \mathrm{~h}$, hasta llegar a un peso constante. Posteriormente, con el contenido de MS se calculó el porcentaje de humedad.

\section{Análisis de azúcares}

Se realizó por el método modificado de Mustafa et al. (2003), para lo cual se tomó $1.0 \mathrm{~g}$ de vainilla molida y se agregaron $60 \mathrm{ml}$ de etanol $80 \%$, y, por ebullición, se concentró a volumen de $20 \mathrm{ml}$; posteriormente, el extracto se filtró y refrigeró por 24 h. Después, se tomó $1 \mathrm{ml}$ de solución y se filtró en cartuchos de limpieza (Cromabond $\mathrm{C}_{18 \mathrm{ec}} 3 \mathrm{ml} \cdot 500 \mathrm{mg}^{-1}, 60 \AA, 45 \mu \mathrm{m}$ ), previamente acondicionados, $\mathrm{y}$, finalmente, se realizó un lavado con $3 \mathrm{ml}$ de agua grado HPLC para asegurar la elución de todos los azúcares. Ambos filtrados se mezclaron y se llevaron a volumen de $5 \mathrm{ml}$; de esta muestra, se filtró $1 \mathrm{ml}$ en acrodisco (Titan, $0.45 \mu \mathrm{m}$ ). El filtrado fue colocado en vial y se analizó por HPLC (High Performance Liquid Chromatography; Series 200, Perkin Elmer ${ }^{\mathrm{TM}}$ ) con automuestreador y detector de índice de refracción. Se utilizó una columna Pinnacle II Amino de $5 \mathrm{~mm} 150 \times 4.6 \mathrm{~mm}$ (RestekTM), y la fase móvil fue una solución de acetonitrilo: agua (80:20, v/v) con tiempo de corrida de $14 \mathrm{~min}$.

Para las curvas de calibración se diluyeron por separado $0.05 \mathrm{~g}$ de fructosa, glucosa y sacarosa a $99.5 \%$ (todos de Sigma-Aldrich, USA) en $10 \mathrm{ml}$ de metanol: agua $(1: 9, \mathrm{v} / \mathrm{v}), \mathrm{y}$ se realizaron las diluciones correspondientes $\left(0.15\right.$ a $\left.5 \mathrm{mg} \cdot \mathrm{ml}^{-1}\right)$. Las condiciones del cromatógrafo fueron $35{ }^{\circ} \mathrm{C}$, flujo de $1 \mathrm{ml} \cdot \mathrm{min}^{-1} \mathrm{y}$ volumen de inyección de $10 \mu \mathrm{L}$. Los resultados se reportaron como porcentaje de azúcares en base seca.

\section{Análisis nutricional}

Se tomaron cuatro frutos de vainilla (verde y beneficiada) por tratamiento, se trituraron y pesaron. El material se secó a $70{ }^{\circ} \mathrm{C}$ durante $48 \mathrm{~h}$ en estufa con circulación forzada de aire (modelo EI35-EA, $\mathrm{NOVATECH}^{\circledR}$, IONC, México); posteriormente, se molió con un molino de cuchillas de acero inoxidable (modelo 38C L10, Thomas Scientific ${ }^{\circledR}$, New Jersey, Estados Unidos), y se tamizó en malla 20. Las muestras se procesaron en los laboratorios de Nutrición y Fertilidad de suelos del Colegio de Postgraduados, Campus Montecillo. Se midió el contenido de macro y micronutrientes de tejidos. El $\mathrm{N}$ se determinó mediante el método de Microkjeldahl (Bremner 1965). El P se midió por colorimetría del complejo fosfo-vanado-molibdato, de acuerdo con el método descrito por la AOAC (1980), con las lecturas respectivas a $470 \mathrm{~nm}$ en un espectrofotómetro (modelo Spectronic 20D, Milton Roy $^{\circledR}$, Colorado, Estados Unidos). Los elementos K, Ca, $\mathrm{Mg}, \mathrm{B}, \mathrm{Cu}, \mathrm{Fe}, \mathrm{Mn}$ y $\mathrm{Zn}$ se analizaron mediante espectrofotómetro de absorción atómica (AES-ICP) (modelo 725-ES, Varian ${ }^{\circledR}$, Mulgrave, Australia).

\section{Análisis estadístico}

Los resultados de las variables se analizaron mediante un diseño completamente al azar. La comparación de medias se calculó con la prueba de Tukey $(\mathrm{P} \leq 0.05)$ y el software Statistical Analysis System (SAS Institute 2002). Para el contenido de azúcares se realizó una gráfica de efectos principales con Minitab (2020).

\section{Resultados y Discusión}

\section{Peso y dimensiones del fruto}

La longitud tanto de los frutos verdes como beneficiados no tuvo diferencias significativas entre la etapa de desarrollo de la semana 20 hasta la 32 DP. Los frutos pueden considerarse de tamaño mediano a pequeño, que, de acuerdo con la NOM-182-SCFI-2011, debe 
ser mayor a $150 \mathrm{~mm}$. Los frutos verdes tuvieron una longitud entre 162 a $167 \mathrm{~mm}$, y después del beneficiado - que implica un proceso de deshidratación- ésta se redujo a 155 a $157 \mathrm{~mm}$. Cervantes et al. (2018) reportan en frutos beneficiados procedentes de San Luis Potosí una longitud entre 108 y $192 \mathrm{~mm}$, mientras que en los frutos del estado de Veracruz de 130 a $220 \mathrm{~mm}$.

Después de la polinización, los frutos de vainilla crecen a diferente velocidad; Exley (2011) menciona que el crecimiento se presenta entre las 8 y 12 SDP, mientras que Van Dyk et al. (2014) mencionan que los frutos crecen paulatinamente a partir de la primera semana después de la polinización, y alcanzan su tamaño final entre las 10 y 15 SDP; posteriormente, no se observan cambios significativos en la apariencia de los mismos, hasta las 32-36 SDP, en donde inicia el amarillamiento y dehiscencia de la parte distal (Frenkel et al. 2011). Esta circunstancia afecta la decisión de los productores al estandarizar un índice de cosecha, por lo que, al no observar cambios en el tamaño ni color del fruto, toman la decisión de cosechar de forma temprana. Sin embargo, después de beneficiar el fruto, notan un rendimiento muy bajo (Cuadro 1); por ejemplo, si se cosecharan $100 \mathrm{~kg}$ de fruto verde de 20 SDP, obtendríamos $12.13 \mathrm{~kg}$ de vainilla beneficiada, mientras que, con frutos de $32 \mathrm{SDP}$ se tendrían 17.95 kg. Esto indica ventajas de cosechar los frutos con mayor edad. No se observaron diferencias entre el peso y las dimensiones de los frutos de diferente edad, tanto en estado verde como beneficiado (Cuadro 1).

\section{Materia seca y humedad}

No se presentaron diferencias significativas en el porcentaje de materia seca en fruto verde de diferente edad (Cuadro 2). Van Dyk et al. (2014), al evaluar el contenido de materia seca en frutos verdes de diferente edad, mostraron que es diferencial dentro de cada fruto, pues existe menor acumulación en la parte proximal del fruto entre la edad de 20-35 SDP ( 12\%), mientras que en la parte media y distal acumula un poco más (12-15\%) en el mismo periodo.

Los resultados indican que el fruto beneficiado con 20 SDP presentaron los valores menores de materia seca. Esta discrepancia podría atribuirse a que el muestreo se llevó a cabo en diferentes partes del fruto, cuya acumulación de materia seca es diferencial, además de que se observa una alta variabilidad entre los mismos frutos, lo que es evidente por el alto coeficiente de variación. En relación con los frutos beneficiados, es notable que el porcentaje de materia seca es significativamente menor en los frutos de menor edad; esto confirma el bajo rendimiento de fruto verde a beneficiado, lo cual se ve reflejado en un fruto más delgado, color ocre y con poco brillo (Figura 1).

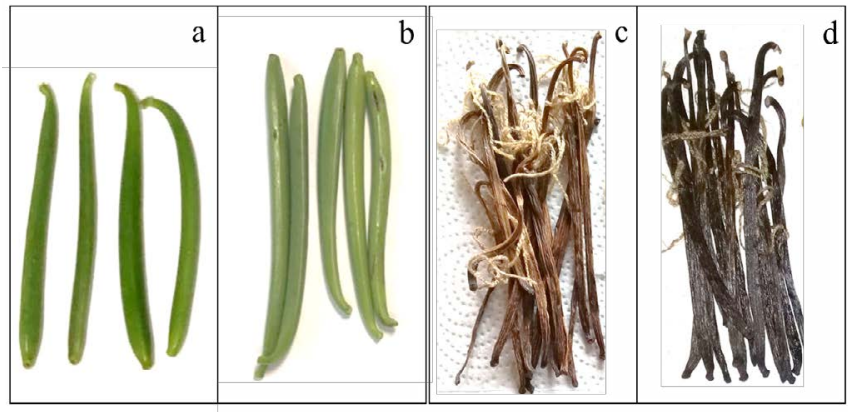

Figura 1. Apariencia de frutos de vainilla de diferente edad: a) fruto verde de $20 \mathrm{SDP}, \mathrm{b})$ fruto verde de $28 \mathrm{SDP}$; c) fruto beneficiado de $20 \mathrm{SDP}$ y d) fruto beneficiado de 32 SDP. SPD: semanas después de polinización.

\section{Contenido de azúcares}

Generalmente, los carbohidratos son los constituyentes más abundantes en los frutos después del agua (Gutiérrez-Bello 2000). En el fruto verde se presenta un contenido total de entre 15.1-25.8\%. Los azúcares como fuente de carbono desempeñan un papel importante en la vainilla como: fuente de energía, precursor de la vainillina y cofactor.

En fruto verde, la sacarosa es el azúcar más abundante con $80 \%$ del total de los azúcares (Sánchez-Galindo et al. 2018). Sin embargo, durante el proceso de beneficio, las proporciones cambian, ya que se presenta un descenso de hasta $95 \%$ del contenido de sacarosa, y un leve incremento en glucosa y fructosa. Ramachandra y Ravishankar (2000) reportan en vainilla beneficiada contenido de azúcares de 70 a $200 \mathrm{~g} \mathrm{~kg}^{-1}$, con la glucosa y fructosa como los componentes principales, y pequeñas cantidades de sacarosa, lo cual hace pensar que la proporción de estos tres azúcares dependerá del tipo de beneficiado al que se somete el fruto. Después del matado con agua caliente, los frutos se colocan en cajones, para limitar la concentración de oxígeno. A medida que las condiciones de anoxia dominan, la población de microorganismos anaeróbicos incrementa y aparecen 
Cuadro 1. Medias de las variables de calidad evaluadas en fruto verde y beneficiado de diferente edad de Vanilla planifolia Jacks. ex Andrews, Mahuajco, Coxcatlán, San Luis Potosí, México.

\begin{tabular}{|c|c|c|c|c|c|c|c|c|c|}
\hline \multirow{2}{*}{$\begin{array}{l}\text { Edad del } \\
\text { fruto } \\
\text { (SDP) }\end{array}$} & \multicolumn{4}{|c|}{ Fruto verde } & \multicolumn{4}{|c|}{ Fruto beneficiado } & \multirow[t]{2}{*}{ Relación V/B } \\
\hline & $\begin{array}{c}\text { Peso } \\
\text { (g) }\end{array}$ & $\underset{(\mathrm{mm})}{\mathrm{L}}$ & $\underset{(\mathrm{mm})}{\mathrm{D}}$ & $\underset{(\mathrm{mm})}{\mathbf{A}}$ & $\begin{array}{c}\text { Peso } \\
\text { (g) }\end{array}$ & $\underset{(\mathrm{mm})}{\mathrm{L}}$ & $\underset{(\mathrm{mm})}{\mathrm{D}}$ & $\underset{(\mathbf{m m})}{\mathrm{A}}$ & \\
\hline 20 & $8.9 \mathrm{a}$ & $162 \mathrm{a}$ & $8.2 \mathrm{a}$ & $9.2 \mathrm{a}$ & $1.08 \mathrm{a}$ & $153 a$ & $2.1 \mathrm{a}$ & $3.9 \mathrm{a}$ & 8.24 \\
\hline 24 & $7.4 \mathrm{a}$ & $163 \mathrm{a}$ & $7.5 \mathrm{a}$ & $9.2 \mathrm{a}$ & $1.08 \mathrm{a}$ & $155 a$ & $2.0 \mathrm{a}$ & $3.7 \mathrm{a}$ & 6.85 \\
\hline 28 & $9.2 \mathrm{a}$ & $164 \mathrm{a}$ & $8.4 \mathrm{a}$ & $9.7 \mathrm{a}$ & $1.40 \mathrm{a}$ & $156 a$ & $1.8 \mathrm{a}$ & $3.7 \mathrm{a}$ & 6.57 \\
\hline 32 & $7.8 \mathrm{a}$ & 167 a & $7.7 \mathrm{a}$ & $12.0 \mathrm{a}$ & $1.40 \mathrm{a}$ & 157 a & $1.8 \mathrm{a}$ & $3.7 \mathrm{a}$ & 5.57 \\
\hline CV (\%) & 27.88 & 5.39 & 15.25 & 41.51 & 32.77 & 4.70 & 25.83 & 12.72 & - \\
\hline DMSH & 2.84 & 1.08 & 1.48 & 5.11 & 0.49 & 0.89 & 0.62 & 0.59 & - \\
\hline
\end{tabular}

CV: coeficiente de variación; DMSH: diferencia mínima significativa honesta. ${ }^{1}$ Medias con la misma letra dentro de cada columna no difieren estadísticamente (Tukey, $\mathrm{P} \leq 0.05)$. SDP: semanas después de polinización; L: longitud; D: diámetro, A: ancho. V: fruto verde; B: fruto beneficiado.

Cuadro 2. Porcentaje de materia seca (MS) y humedad (H) en frutos verdes y beneficiados de diferente edad de Vanilla planifolia Jacks. ex Andrews.

\begin{tabular}{ccccc}
\hline Semanas después de polinización (SDP) & \multicolumn{2}{c}{ Fruto verde } & \multicolumn{2}{c}{ Fruto beneficiado } \\
\cline { 2 - 5 } & MS & H & MS & H \\
\hline 20 & $11.4 \mathrm{a}$ & $88.6 \mathrm{a}$ & $72.2 \mathrm{~b}$ & $27.8 \mathrm{a}$ \\
24 & $12.4 \mathrm{a}$ & $87.6 \mathrm{a}$ & $80.8 \mathrm{a}$ & $19.2 \mathrm{~b}$ \\
28 & $10.6 \mathrm{a}$ & $89.4 \mathrm{a}$ & $84.2 \mathrm{a}$ & $15.8 \mathrm{~b}$ \\
32 & $12.6 \mathrm{a}$ & $87.4 \mathrm{a}$ & $85.0 \mathrm{a}$ & $15.0 \mathrm{~b}$ \\
\hline CV (\%) & 21.39 & 2.84 & 3.22 & 13.33 \\
DMSH & 4.72 & 4.72 & 4.87 & 4.87 \\
\hline
\end{tabular}

CV: coeficiente de variación; DMSH: diferencia mínima significativa honesta. Medias con la misma letra dentro de la columna no difieren estadísticamente (Tukey, P $\leq 0.05)$. MS: materia seca, H: humedad.

las moléculas asociadas con la anaerobiosis, sobre todo aquellas relacionadas con la transformación glucolítica (glucosa a piruvato, y posteriormente a acetaldehído y etanol).

El contenido de sacarosa disminuyó de fruto verde a beneficiado en todas las fechas evaluadas. El fruto verde de 28 SDP tuvo un contenido significativamente mayor de sacarosa en relación con los frutos de las otras edades. En fruto beneficiado la sacarosa disminuyó drásticamente, sin diferencias significativas entre semanas (Figura 2). Sánchez-Galindo et al. (2018) reportan una disminución de $46 \%$ de sacarosa en frutos de 32 SDP de fruto verde a beneficiado procedentes de Veracruz, con un aumento significativo en el contenido de fructosa y glucosa. Palama et al. (2009) encontraron en fruto verde cosechado a 32 semanas mayor contenido de glucovanilina, vainillina, glucósido de $p$-hidroxibenzaldehído, $p$-hidroxibenzaldehído y sacarosa. En nuestro estudio, la sacarosa fue mayor que la fructosa y la glucosa para fruto verde en todas las semanas de evaluación, pero menor en fruto beneficiado. Cervantes et al. (2018) reportan la misma condición en fruto beneficiado procedente de San Luis Potosí, donde fructosa y glucosa superan en 18 y $76 \%$ a sacarosa. Durante el proceso de beneficio, se llevan a cabo operaciones cíclicas de exposición al Sol, hasta alcanzar $45^{\circ} \mathrm{C}$, y empacado en cajones para el sudado. Estos pasos permiten la activación del metabolismo anaeróbico, lo cual facilita la proliferación de microorganismos anaeróbicos y la aparición de moléculas asociadas con la anaerobiosis. Además, estas condiciones por periodos cortos de hipoxia estimulan la transformación glucolítica de glucosa en piruvato, que puede formar acetaldehído y etanol. Ambos compuestos también en baja concentración son volátiles y están presentes en el aroma de la vainilla, 

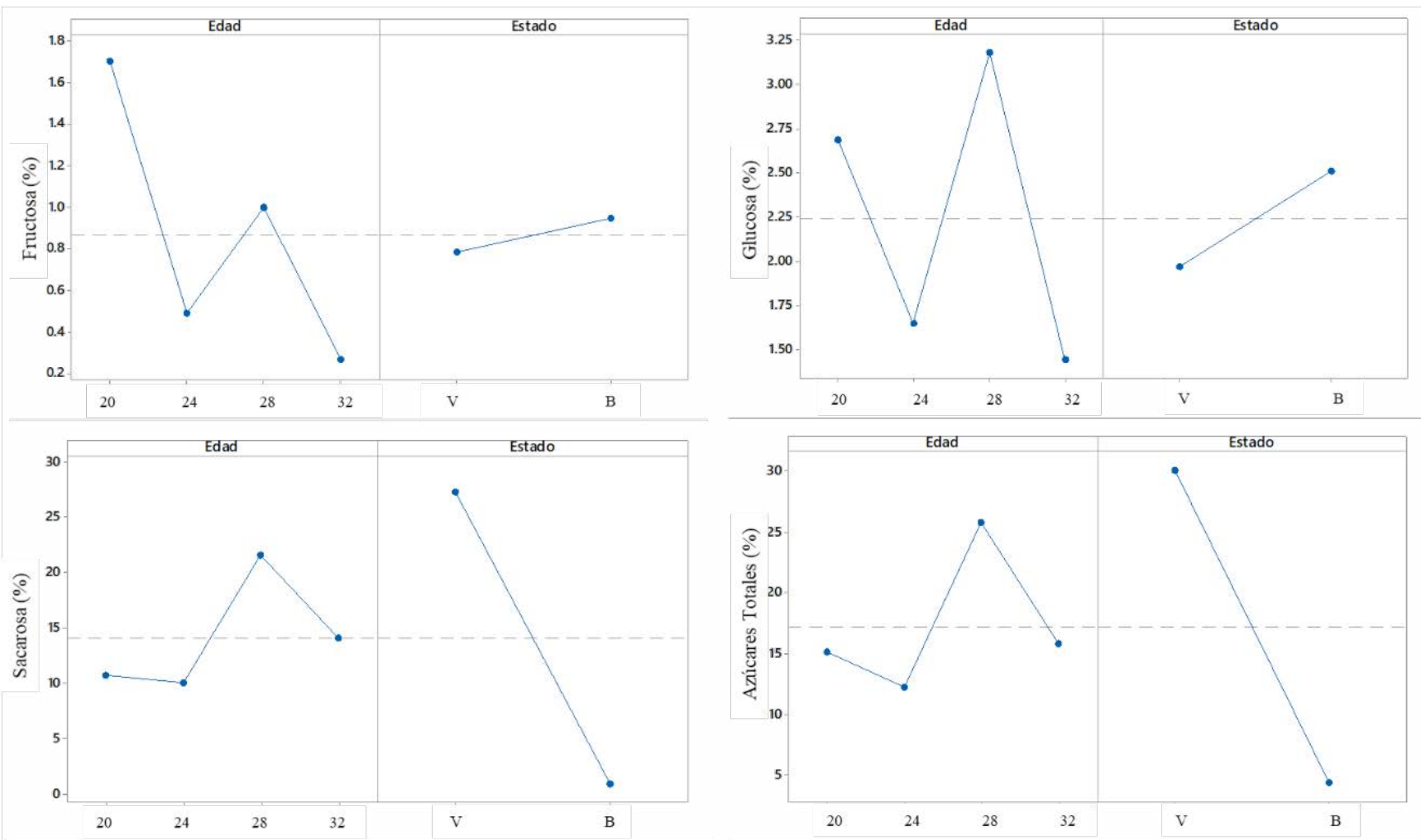

Figura 2. Efectos principales en el cambio de la concentración de azucares (fructosa, glucosa, sacarosa y totales) en frutos de vainilla cosechados a la edad de 20, 24, 28 y 32 SDP en estado verde y beneficiado (V y B).

lo que muestra el papel tan importante de los azúcares en el proceso de beneficio.

Un aspecto relevante es que durante el curado intervienen diferentes especies de hongos endófitos que pueden utilizar el azúcar como sustrato y afectar el sabor de la vainilla beneficiada (Gu et al. 2015). Por ejemplo, la sacarosa es más ampliamente utilizada por hongos como Pestalotiopsis microspora, Diaporthe phaseolorum, Nemania bipapillata, F. oxysporum y F. proliferatum; sin embargo, estos dos últimos también pueden utilizar la fructosa como sustrato (Khoyratty et al. 2015; Moraes et al. 2018; Jian et al. 2019). Se ha informado que ciertos hongos endófitos producen metabolitos secundarios que antes se pensaba que eran producidos por la planta (Giménez et al. 2007).

\section{Análisis nutrimental}

Los elementos más importantes para el crecimiento de las plantas se clasifican, según su concentración, en: macronutrientes primarios (nitrógeno, fósforo y potasio), secundarios (calcio, magnesio) y micronutrientes (azufre, hierro, manganeso, boro, zinc y cobre) (Blume et al. 2010; Clarkson y Hanson 1980).
Al llevar a cabo el análisis nutricional en fruto verde y beneficiado de las cuatro edades, se observó que las concentraciones de macronutrientes incrementaron con la edad del fruto; el mismo comportamiento se encontró con los micronutrientes (Cuadro 3). La concentración de estos elementos aumentó a lo largo del periodo de evaluación, para llegar al máximo a la cosecha. Esto indica que la demanda de nutrientes de los frutos de vainilla se incrementa en porcentaje conforme avanza la etapa de desarrollo de los frutos, y puede relacionarse con la acumulación de glucósidos precursores del aroma que inicia a partir de 15 SDP (Van Dyk et al. 2014).

El $\mathrm{K}$ fue el macronutriente con mayor porcentaje en la primera semana de evaluación con 1.01\% en 20 SDP, seguido de Ca y $\mathrm{N}$, pero se situó en segundo lugar a partir de 24 SDP, con $1.15 \%$, y nuevamente incrementó a las $28 \mathrm{SDP}$, con $1.17 \%$, para alcanzar su máximo a las 32 SDP con $1.22 \%$. En café (Coffea sp.) se ha observado que, durante el periodo del desarrollo de los frutos, el contenido de $\mathrm{K}$ es más alto que el de $\mathrm{N}$, especialmente a partir de los 90 o los 120 días después del día pico de la floración (Sadeghian et al. 2012). Durante el periodo de desarrollo del fruto de vainilla, desde las primeras 
Cuadro 3. Análisis nutrimental de fruto verde y beneficiado de vainilla (macro y micronutrientes)

\begin{tabular}{|c|c|c|c|c|c|c|c|c|}
\hline \multirow[b]{3}{*}{ Macronutrientes (\%) } & \multicolumn{8}{|c|}{ Edad a cosecha (SDP) } \\
\hline & \multicolumn{4}{|c|}{ Fruto verde } & \multicolumn{4}{|c|}{ Fruto beneficiado } \\
\hline & 20 & 24 & 28 & 32 & 20 & 24 & 28 & 32 \\
\hline $\mathrm{N}$ & 0.93 & 1.00 & 1.07 & 1.12 & 1.70 & 1.68 & 1.75 & 1.82 \\
\hline $\mathrm{P}$ & 0.06 & 0.07 & 0.11 & 0.15 & 0.10 & 0.08 & 0.17 & 0.23 \\
\hline K & 1.01 & 1.15 & 1.17 & 1.22 & 2.11 & 1.94 & 2.73 & 3.12 \\
\hline $\mathrm{Ca}$ & 0.98 & 1.19 & 1.35 & 1.47 & 2.90 & 2.06 & 1.90 & 1.56 \\
\hline $\mathrm{Mg}$ & 0.04 & 0.07 & 0.10 & 0.12 & 0.40 & 0.33 & 0.30 & 0.28 \\
\hline \multicolumn{9}{|l|}{ Micronutrientes (mg kg-1) } \\
\hline B & 19.04 & 22.15 & 23.15 & 24.36 & 23.19 & 23.15 & 30.15 & 35.95 \\
\hline $\mathrm{Cu}$ & 4.13 & 5.9 & 6.12 & 7.16 & 7.10 & 9.12 & 7.20 & 8.57 \\
\hline $\mathrm{Fe}$ & 14.2 & 19.12 & 19.27 & 19.54 & 34.10 & 33.00 & 30.12 & 28.01 \\
\hline Mn & 20.15 & 26.30 & 26.95 & 27.84 & 25.30 & 37.50 & 46.19 & 56.86 \\
\hline $\mathrm{Zn}$ & 14.17 & 15.01 & 16.4 & 17.64 & 24.40 & 24.20 & 24.00 & 23.97 \\
\hline
\end{tabular}

SDP = semanas después de la polinización.

semanas posteriores a la polinización hasta 32 SDP, la demanda de K es alta, similar a otros cultivos, pues el K participa en la activación de numerosas enzimas útiles en la fotosíntesis, síntesis de proteínas y carbohidratos. Al participar en estos procesos metabólicos, el K actúa favoreciendo el crecimiento vegetativo, la fructificación, la maduración y la calidad de los frutos. Diez et al. (2016), al evaluar el efecto de la dosis y el tipo de fertilizante sobre la floración y fructificación de las plantas de vainilla, reportaron que existe mayor contenido de NPK en ramas vegetativas que en ramas reproductivas; esta respuesta se atribuye a la alta demanda de nutrientes para la formación de flores y frutos. Sin embargo, la información sobre el contenido de nutrientes en frutos de vainilla es escasa, por lo que nuestro estudio contribuye al conocimiento en esta área.

El Ca, aunque se considera un macronutriente secundario (Blume et al. 2010), mantuvo un incremento constante conforme avanzaba la etapa de desarrollo del fruto de vainilla y mostró concentraciones más altas en las últimas tres semanas de evaluación, para alcanzar su máximo porcentaje en 32 SDP con $1.47 \%$. El Ca mantiene la estructura de la pared celular, debido a su capacidad para servir como puente entre las sustancias pécticas tanto de la pared celular como de la lámina media, gracias a lo cual forma pectato cálcico, que aporta estructura al tejido y evita el ablandamiento (Alandes et al. 2006).
En fruto verde, la concentración de $\mathrm{N}$ también incrementó conforme avanzó la etapa de desarrollo del fruto, para alcanzar el máximo porcentaje a 32 SDP, con $1.12 \%$, por lo que se ubicó en el tercer sitio, después de Ca y K. El N participa directamente en la síntesis de aminoácidos, proteínas, ácidos nucleicos y otros constituyentes celulares necesarios para el desarrollo del fruto (Stitt 1999).

Los micronutrientes son elementos fundamentales en el crecimiento y desarrollo de los frutos, pues actúan como cofactores de enzimas, toman parte en procesos metabólicos y reacciones redox, así como de transferencia de electrones. La deficiencia de estos micronutrientes afecta fuertemente la producción de metabolitos, lo cual es particularmente importante en los frutos de vainilla, cuya calidad como producto beneficiado depende de la cantidad de metabolitos secundarios (Bhat et al. 2020). El micronutriente con mayor concentración fue $\mathrm{Mn}$ durante el periodo máximo de evaluación alcanzado (27.84 $\left.\mathrm{mg} \mathrm{kg}^{-1}\right)$ en 32 SDP. El Mn actúa como co-factor para varias enzimas fundamentales en la biosíntesis de los metabolitos secundarios de la planta asociados con la vía de ácido shiquímico, incluyendo aminoácidos aromáticos fenólicos, cumarinas, ligninas y flavonoides, cuyos niveles son bajos en casos con deficiencias de $\mathrm{Mn}$ (Römheld y Kirkby 2010). El Ca y Mn incrementaron conforme avanzó la edad del fruto con $1.47 \%$ y 27.84 $\mathrm{mg} \mathrm{kg}^{-1}$, respectivamente, en la semana 32. De la misma 
manera, incrementó la concentración de los micronutrientes $\mathrm{B}, \mathrm{Fe}, \mathrm{Zn}, \mathrm{y} \mathrm{Cu}$.

Para el beneficiado, los frutos verdes fueron sometidos al proceso de matado (escaldado), consistente en la inmersión del fruto en agua caliente a $65-70{ }^{\circ} \mathrm{C}$ por 2 o $3 \mathrm{~min}$; bajo estas condiciones existe interrupción de la función respiratoria y ruptura de la membrana celular, lo cual permite que las enzimas entren en contacto con los sustratos, para iniciar diferentes reacciones que resultan en la producción de compuestos del aroma y sabor (Ramachandra y Ravishankar 2000).

Durante el proceso de beneficio se presentan cambios significativos en la coloración de los frutos, como resultado de la actividad de la polifenoloxidasa. Esta enzima contiene cobre y cataliza la reacción entre un grupo fenol y oxígeno, lo que produce quinonas, compuestos responsables de la pigmentación amarilla-marrón del tejido vegetal (Martínez-Valverde et al. 2000).

En fruto beneficiado, el Ca fue el elemento de mayor concentración, el cual disminuyó con la edad de $2.90 \%$ a las 20 SDP hasta $1.56 \%$ a las 32 SDP. Esta disminución podría relacionarse con el efecto que tuvo el escaldado del fruto, el cual produce la ruptura de la membrana celular y causa ablandamiento del tejido, asociado a modificaciones que ocurren en los polímeros de la pared celular (Angeletti et al. 2010) y a un conjunto de enzimas que contribuyen a los cambios en la pared y membrana celular, tales como: poligalacturonasa (PG), pectato liasa, pectina metilesterasa, $\beta$-galactanasa / $\beta$-galactosidasa ( $\beta$-Gal) y $\alpha$-arabinasa/ $\alpha$-arabinofuranosidasa (Rose et al. 2003); éstas contribuyen a la pérdida de firmeza, lo que es muy evidente en los frutos beneficiados.

K fue el segundo en importancia después de Ca: a las 28 y 32 SDP fue el elemento más abundante (2.73 y $3.12 \%$, respectivamente). Ramírez et al. (2012), al evaluar pulpa de guanábana (Annona muricata L.) y escaldada a $70{ }^{\circ} \mathrm{C}$ por $20 \mathrm{~min}$, observaron mayor concentración de $\mathrm{K}$. El $\mathrm{N}$ fue el tercer elemento de importancia, aunque no se tienen reportes sobre la forma en la que se encuentra presente en vainilla durante el proceso de beneficiado, pero se ha documentado la importancia este elemento en la fermentación del mosto de uva, en la forma de aminoácidos y amonio, pues resulta esencial para el desarrollo de la actividad microbiológica durante la fermentación alcohólica. Las necesidades de nitrógeno se acentúan cuando la concentración de azúcares de las uvas es alta, ya que hacen falta más generaciones de levaduras para consumirlos en su totalidad, por lo que la demanda de nitrógeno es mayor (Gobert et al. 2019).

Con relación a los micronutrientes, el Mn fue el de mayor acumulación, con un máximo (56.86 mg kg-1) en 32 SDP. Su importancia radica en la contribución como co-factor para varias enzimas y biosíntesis de aminoácidos aromáticos, compuestos fenólicos, cumarinas, ligninas y flavonoides, cuyos niveles son bajos en casos con deficiencias de Mn (Römheld y Kirkby 2010). El B incrementó en las dos últimas semanas de evaluación, para alcanzar un máximo (35.95 mg kg-1) en 32 SDP. El Cu fue el micronutriente con menor concentración en el fruto beneficiado, con un máximo de $8.57 \mathrm{mg} \mathrm{kg}^{-1}$ a las 32 SDP. Sin embargo, su importancia durante el proceso de beneficiado puede radicar en la actividad de la polifenoloxidasa, enzima que contiene cobre y cataliza la reacción entre un grupo fenol y el oxígeno, lo que da como resultado la formación de quinonas, responsables de la coloración marrón de los frutos (Martínez-Valverde et al. 2000).

El contenido total de macro y micronutrientes incrementó conforme avanzó la edad del fruto, llegando a un máximo a las 32 SDP (Figura 3).

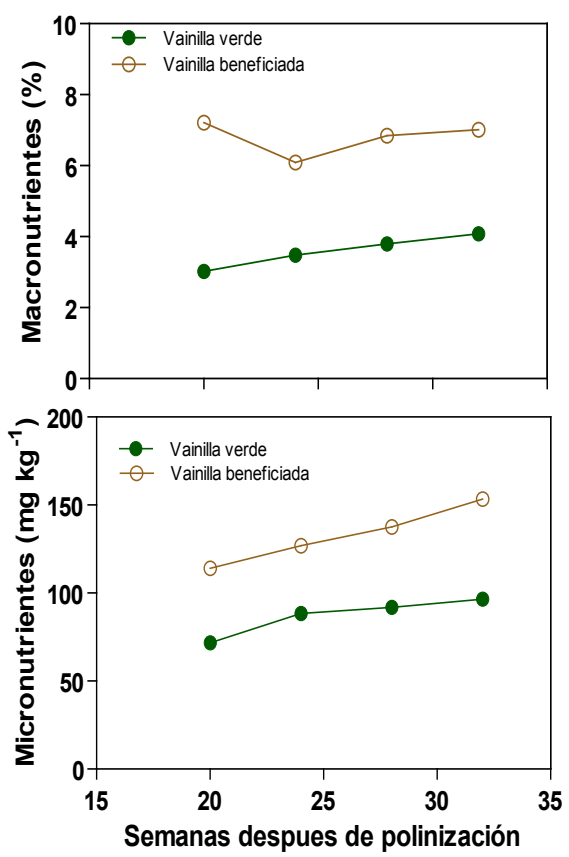

Figura 3. Contenido total de macro y micronutrientes en frutos de vainilla verde y beneficiado a diferentes edades de fruto. 
Ordóñez-Araque y Pardo-Yoza (2018) observaron en Borojoa patinoi Cuatrec (Borojó), un incremento en el contenido de minerales después de un proceso de deshidratación, por lo que el proceso de beneficio, al contemplar procesos térmicos y de fermentación, contribuye al aumento de estos elementos.

\section{Coclusiones}

Se observó que los frutos de vainilla de 20 SDP reducen su peso 8.24 veces de estado verde a beneficiado, mientras que esta reducción es de 5.57 en frutos de 32 SDP, lo que repercute en el rendimiento y la calidad del fruto. El contenido de azúcares totales en fruto verde muestra un incremento sustancial a la semana $28 \mathrm{y}$ desciende significativamente para la 32. La sacarosa es el principal azúcar del fruto verde (80\%), con pequeñas cantidades de glucosa y fructosa, que incrementan levemente en fruto beneficiado debido a la hidrólisis de sacarosa. El contenido de macro y micronutrientes aumentó conforme avanzó la edad del fruto; los macronutrientes con mayor concentración fueron el Ca y el K, mientras que el Mn fue el principal micronutriente. Los resultados de este estudio muestran la importancia de cosechar los frutos de vainilla después de las 28 SDP. 


\section{Literatura Citada}

Alandes L, Hernando I, Quiles A, Pérez MI, Lluch MA. 2006. Cell wall stability of fresh-cut Fuji apples treated with calcium lactate. Journal of Food Science 71: S615-S620. https://doi.org/10.1111/j.1750-3841.2006.00180.x

Angeletti P, Castagnasso H, Miceli E, Terminiello L, Concellón A, Chaves A, Vicente AR. 2010. Effect of preharvest calcium applications on postharvest quality, softening and cell wall degradation of two blueberry (Vaccinium corymbosum) varieties. Postharvest Biology and Technology 58: 98-103. https://doi.org/10.1016/j. postharvbio.2010.05.015

[AOAC]. Association of Official Analytical Chemist. 1980. Official Methods of Analysis. Association of Official Analytical Chemist. Washington, Estados Unidos.

Bhat BA, Islam ST, Ali A, Sheikh BA, Tariq L, Islam SU, Hassan DTU. 2020. Role of micronutrients in secondary metabolism of plants. En: Aftab T, Hakeem KR, editores. Plant Micronutrients. Nueva York, Springer. P. 311-329. https://doi.org/10.1007/978-3-030-49856-6_13

Blume H-P, Brümmer GW, Fleige H, Horn R, Kandeler E, Kögel-Knabner I, Kretzschmar R, Stahr K, Wilke B-M. 2010. Scheffer/Schachtschabel Soil Science. Springer, Alemania.

Bory S. 2007. Diversité de Vanilla planifolia G. Jackson dans I ocean Indien et de ses especies apparentées; aspects genétiques, cytogénetiques et epigénétiques. Tesis de Doctorado en Ciencias. Université de la Réunion. Réunion. https://doi.org/10.13140/RG.2.1.1156.2408

Bremner JM. 1965. Inorganic forms of nitrogen. En: Norman AG, editor. Methods of Soil Analysis: Part 2 Chemical and Microbiological Properties. Madison, Estados Unidos.

Brillouet JM, Odoux E, Conejero G. 2010. A set of data on green, ripening and senescent vanilla pod (Vanilla planifolia; Orchidaceae): Anatomy, enzymes, phenolics and lipids. Fruits 65: 221-235. https://doi.org/10.1051/ fruits/2010018

Cervantes CA, Lima MM, Delgado AA, Herrera CBE, Arévalo GGA, Soto HRM, Arévalo GML. 2018. Calidad de frutos vainilla (Vanilla planifolia Jacks. ex Andrews) procedente de la Huasteca Potosina, México. Nova Scientia 10: 360-378. https://doi. org/10.21640/ns.v10i21.1586

Clarkson DT, Hanson JB. 1980. The mineral nutrition of higher plants. Annual Review of Plant Physiology 31: 239-298. https://doi.org/10.1146/annurev. pp.31.060180.001223

Diez MC, Osorio NW, Moreno F. 2016. Effect of dose and type of fertilizer on flowering and fruiting of vanilla plants. Journal of Plant Nutrition 39: 1297-1310. https:// doi.org/10.1080/01904167.2015.1098673

Dignum MJ, van der Heijden R, Kerler J, Winkel C, Verpoorte R. 2004. Identification of glucosides in green beans of Vanilla planifolia Andrews and kinetics of vanilla $\beta$-glucosidase. Food Chemistry 85: 199-205. https:// doi.org/10.1016/S0308-8146(03)00293-0

Dunphy P, Bala K. 2011. Green vanilla bean quality. Perfumer \& Flavorist 36: 38-46.

Exley R. 2011. Vanilla production in Australia. En: HavkinFrenkel D, Belanger F, editores. Handbook of Vanilla Science and Technology. Hoboken, Wiley-Blackwell. P. 69-78.

Font-Quer P. 1953. Diccionario de botánica. Labor. Barcelona, España.

Fouché JG, Jouve L. 1999. Vanilla planifolia: History, botany and culture in Reunion island. Agronomie 19: 689-703.

Frenkel C, Ranadive AS, Vázquez JT, Havkin-Frenkel D. 2011. Curing of vanilla. En: Havkin-Frenkel D, Belanger FC, editores. Handbook of Vanilla Science and Technology. Hoboken, Wiley Blackwell. P. 191-221.

García E. 2005. Modificación al sistema de clasificación climática de Köppen. Universidad Nacional Autónoma de México. Distrito Federal, México.

Giménez C, Cabrera R, Reina M, González-Coloma A. 2007. Fungal endophytes and their role in plant protection. Current Organic Chemistry 11: 707-720. https:// doi.org/10.2174/138527207780598765

Gobert A, Tourdot-Maréchal R, Sparrow C, Morge C, Alexandre H. 2019. Influence of nitrogen status in wine alcoholic fermentation. Food Microbiology 83: 71-85. https://doi.org/10.1016/j.fm.2019.04.008

Gu F, Chen Y, Fang Y, Wu G, Tan L. 2015. Contribution of Bacillus isolates to the flavor profiles of vanilla beans assessed through aroma analysis and chemometrics. Molecules 20: 18422-18436. https://doi.org/10.3390/ molecules201018422

Gutiérrez-Bello J. 2000. Ciencia bromatológica: principios generales de los alimentos. Ediciones Díaz de Santos. p. 3-26.

Havkin-Frenkel D, French JC, Graft NM, Joel DM, Park FE, Frenkel C. 2004. Interrelation of curing and botany in vanilla (Vanilla planifolia) bean. Acta Horticulturae 629: 93-102. https://doi.org/10.17660/ActaHortic.2004.629.12 
Hernández J, Lubinsky P. 2011. Cultivation systems. En: Odoux E, Grisoni, M, editores. Vanilla. Medicinal and Aromatic Plants-Industrial Profiles. Boca Ratón, CRC Press. P. 75-95.

Hooker L. 2017. Qué país es el mayor productor del "oro rojo" más caro del mundo. BBC News Mundo. [citado 2021, julio 3]. Disponible en: https://www.bbc.com/ mundo/noticias-41261244

Jian Q, Li T, Wang T, Zhang Y, Zhao Z, Zhang X, Gong L, Jiang Y. 2019. New insights into fumonisin production and virulence of Fusarium proliferatum underlying different carbon sources. Food Research International 116: 397-407. https://doi.org/10.1016/j.foodres.2018.08.053

Khoyratty S, Dupont J, Lacoste S, Palama TL, Choi YH, Kim HK, Payet B, Grisoni M, Fouillaud, Verpoorte, R, Kodja H. 2015. Fungal endophytes of Vanilla planifolia across Réunion Island: Isolation, distribution and biotransformation. BMC Plant Biology 15: 1-19. https:/doi. org/10.1186/s12870-015-0522-5

Martínez-Valverde I, Periago MJ, Ros G. 2000. Significado nutricional de los compuestos fenólicos de la dieta. Archivos Latinoamericanos de Nutrición 50: 5-18.

Minitab. 2020. Introducción al Minitab Statistical Software. [citado 2020 septiembre 1]. Disponible en: https://www. minitab.com/content/dam/www/en/uploadedfiles/documents/getting-started/MinitabGettingStarted_ESMX. pdf

Moraes AC, Costa-Rodrigues A, Lopes-Santos Silva L, Machado-Gois de Oliveira LJ, Silva-Sardinha DH, Gomes da Costa M. 2018. Morphological aspects and effect of carbon sources in the physiology of Fusarium oxysporum f. sp. passiflorae. Emirates Journal of Food and Agriculture 30: 77-84. https://doi.org/10.9755/ ejfa.2018.v30.i1.1588

Mustafa K, Mustafa E, Mustafa KU, Mehmet A. 2003. Comparison of different extraction and detection methods for sugars using amino-bonded phase HPLC. Journal of Chromatographic Science 41: 331-333. https:// doi.org/10.1093/chromsci/41.6.331

Núñez-Torrón SA. 2020. Hierbas a precio de oro: las 5 especias más caras del mundo. TICbeat. [citado 2020 agosto 18]. Disponible en: https://www.ticbeat.com/cyborgcultura/hierbas-a-precio-de-oro-las-5-especias-mas-caras-del-mundo/

Odoux E, Brillouet JM. 2009. Anatomy, histochemistry and biochemistry of glucovanillin, oleoresin and mucilage accumulation sites in green mature vanilla pod
(Vanilla planifolia; Orchidaceae): A comprehensive and critical reexamination. Fruits 64: 221-241. https://doi. org/10.1051/fruits/2009017

Ordóñez-Araque R, Pardo-Yoza L. 2018. Cuantificación de hierro, calcio y fósforo en procesos térmicos aplicados al borojó (Borojoa patinoi Cuatrec). IDESIA 36: 275-281. https://doi.org/10.4067/S0718-34292018005000802

Palama TL, Khatib A, Choi YH, Payet B, Fock I, Verpoorte R, Kodja H. 2009. Metabolic changes in different developmental stages of Vanilla planifolia pods. Journal of Agricultural and Food Chemistry 57: 7651-7658. https:// doi.org/10.1021/jf901508f

Ramachandra RS, Ravishankar GA. 2000. Vanilla flavour: Production by conventional and biotechnological routes. Journal of the Science of Food and Agriculture 80: 289-304. https://doi.org/10.1002/1097-0010(200002)8 0:3<289::AID-JSFA543>3.0.CO;2-2

Ramírez MR, De Moreno LA, Acosta K, Yamarte M, Sandoval L. 2012. Efecto del escaldado sobre la calidad nutricional de pulpa de guanábana (Annona muricata L.). Revista Iberoamericana de Tecnología Postcosecha 13: 48-57.

Römheld V, Kirkby EA. 2010. Research on potassium in agriculture: Needs and prospects. Plant and Soil 335: 155180. https://doi.org/10.1007/s11104-010-0520-1

Rose JKC, Catalá C, Gonzalez CC, Zinnia H, Roberts J. 2003. Plant cell wall disassembly. The Plant Cell Wall. Oxford Blackwell Publishing. Hoboken, Estados Unidos.

Sadeghian S, Mejía B, González H. 2012. Acumulación de nitrógeno fósforo y potasio en los frutos de café. Revista Cenicafé 63: 7-18.

Sánchez-Galindo M, Arévalo-Galarza ML, DelgadoAlvarado A, Herrera-Cabrera BE, Osorio-García C. 2018. Calidad de fruto verde y beneficiado de vainilla (Vanilla planifolia Jacks. ex Andrews) con relación a su edad a la cosecha. Revista Chapingo. Serie Horticultura 24: 203-213. https://doi.org/10.5154/r.rchsh.2018.02.004

SAS Institute. 2002. Statistical Analysis System. SAS/STAT user's guide version 9.0. Cary, Estados Unidos.

[SE]. Secretaría de Economía. 2011. Norma Oficial Mexicana NOM-182-SCFI-2011, Vainilla de Papantla, extractos y derivados-Especificaciones, información comercial y métodos de ensayo (prueba). [citado 2020 agosto 18]. Disponible en: http://www.dof.gob.mx/normasOficiales/4477/seeco/seeco.htm

[SMN]. Servicio Meteorológico Nacional. 2019. Reporte Anual. Comisión Nacional del Agua. Ciudad de México, México. 
Stitt M. 1999. Nitrate regulation of metabolism and growth.

Current Opinion in Plant Biology 2: 178-186. https://doi. org/10.1016/S1369-5266(99)80033-8

Van Dyk S, Holford P, Subedi P, Walsh K, Williams M, McGlasson WB. 2014. Determining the harvest maturity of vanilla beans. Scientia Horticulturae 168: 249-257. https://doi.org/10.1016/j.scienta.2014.02.002

Xochipa-Morante RC, Delgado-Alvarado A, HerreraCabrera BE, Escobedo-Garrido JS, Arévalo-Galarza ML. 2016. Influencia del proceso de beneficiado tradicional mexicano en los compuestos del aroma de Vanilla planifolia Jacks. ex Andrews. Agroproductividad 9: 56-62. 